\title{
NON-TARGETED EFFECTS OF FACTORS FROM BLOOD SERUMS OF CHERNOBYL POPULATIONS*
}

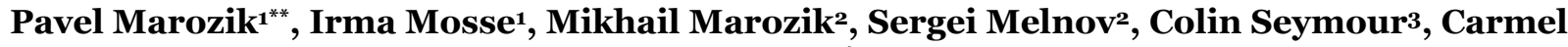 Mothersil3}

\author{
${ }^{1}$ Institute of Genetics \& Cytology NAS Belarus, Minsk, Belarus \\ 2International Environmental Sakharov University, Minsk, Belarus \\ 3McMaster University, Hamilton, Canada
}

\begin{abstract}
In the present work, the phenomenon of bystander effects (transfer of damaging factors from affected cells to intact cells) induced in vivo has been studied. A newly developed method allows evaluation of the effects of bystander factors from sera of different groups of populations on immortalized culture of human keratinocytes. This method has serious advantages as compared to that which was used earlier. The level of damaging bystander factors in blood of people irradiated by the Chernobyl accident was estimated. Affected population groups included liquidators of the Chernobyl accident and people living and working in areas of Gomel region contaminated by radionuclides. The data clearly shows that blood serum samples of irradiated populations contain some kind of factors, induced in vivo and able to cause cell damage when transferred to in vitro conditions. These factors are able to circulate in blood stream for more than 20 years.
\end{abstract}

Key words: bystander effects, clastogenic factors, human keratinocytes, Chernobyl liquidators, affected population.

DOI: $10.21175 / \operatorname{RadJ} .2016 .01 .010$

\section{INTRODUCTION}

Because of human activities, involving the use of naturally occurring or artificially produced radioactive substances, radioactive materials contaminate huge territories of our Planet. Radioactive pollutants affect soil, water, air. Such atmospheric pollution presents a hazard not only to those engaged in work but also the public. As a result, millions of people live and work under the conditions of chronic low dose radiation exposure.

There are various possible biological effects of ionizing radiation on human health. However, from the population viewpoint, the most serious are genetic effects, which are able to transfer from generation to generation and accumulate as a genetic load.

Ever since Roentgen made the discovery of X-rays more than a hundred years ago, it had been generally accepted that the important biological effects of ionizing radiation in mammalian cells were a direct consequence of unrepaired or misrepaired DNA damage in the irradiated cells. The classical dogma of radiation biology, as narrowly interpreted from target theory, asserts that genetic damage occurs only during or very shortly after deposition of energy in nuclear DNA (targeted effects) and, is due only to the direct action of the irradiation or from very short lived oxyradicals generated by it, and that the course of biological consequences is fixed within one or two cell generations.
However, recent experimental evidence, mainly from in vitro $\alpha$-particle studies, indicates that ionizing radiation can cause biological effects, including DNA damage, by mechanisms that are independent of nuclear traversals - "non-targeted" effects. Several studies have shown that genetic changes occur in a greater number of cells than expected when mammalian cell cultures are exposed to low fluencies of $\alpha$-particles by which only a very small fraction of the cells is traversed by a particle track and thus directly exposed to radiation [1-3]. These studies, along with others involving low linear energy transfer radiation from incorporated radionuclides [4] and the transfer of growth media from irradiated to non-irradiated cell cultures [5], and microbeam studies [6] challenge the paradigm that radiation traversal through the nucleus of a cell is a prerequisite to produce genetic changes or a biological response. They indicate that cells located near directly irradiated cells or recipients of medium from irradiated cultures can also respond to the radiation exposure. These evidence suggests the existence of a phenomenon of information transfer from irradiated cells to non-irradiated (so-called radiation induced bystander effect, RIBE).

Such bystander factors could be induced not only by ionizing radiation, but also observed under different pathological conditions [7]. The specific nature of these factors and mechanism of RIBE are still unknown [8].

In recent years some evidence of RIBE induced in vitro was obtained, while the effects in vivo are not enough studied.

\footnotetext{
* The paper was presented at the Third International Conference on Radiation and Applications in Various Fields of Research (RAD 2015), Budva, Montenegro, 2015.

${ }^{* *}$ Contact: P.Marozik@igc.bas-net.by
} 
Effects of bystander factors induced in vivo in blood of people affected by the Chernobyl accident are of particular interest.

For the first time such studies were performed by Dr. I. Emerit [9], when it was shown that blood sera from liquidators of the Chernobyl accident or residents of areas contaminated by radionuclides contained an increased level of bystander factors. These factors increased the frequency of chromosome aberrations and micronuclei in own lymphocytes of serum donors even decades since irradiation.

In Emerit studies own lymphocytes of serum donors were used as a test-system. However, these cells were already exposed to ionizing radiation; as a result, the level of chromosome aberrations and micronuclei was increased there. At the same time, human peripheral blood lymphocytes under in vitro conditions could be subcultured only for 2-4 days, therefore it is impossible to evaluate serum effects for a longer period.

In the present study, a new method was developed which allows evaluation of the effects of bystander factors from sera of different groups of populations on immortalized culture of human keratinocytes. This method has serious advantages as compared to that which was used earlier: cells could be cultivated for a longer period of time (re-cultivation once a week), they are more sensitive as compared to human peripheral blood lymphocytes, and experiments could be easily repeated, allowing simple comparison and interpretation of the obtained results.

Using this method, we evaluated the level of damaging bystander factors in blood of populations affected by the Chernobyl accident.

According to the most wildly accepted estimates, the most affected groups of population are the following:

- accident liquidators - hundreds of thousands of workers and military personnel, who were involved in the emergency actions on the site during the accident and the subsequent clean-up operations which lasted for a few years;

- evacuees from dangerously contaminated territories inside the $30-\mathrm{km}$ zone around the power plant;

- people living and working in contaminated areas of the former Soviet Union;

- populations outside the former Soviet Union the radioactive materials of a volatile nature (such as iodine and cesium) that were released during the accident spread throughout the entire Northern hemisphere. The doses received by populations outside the former Soviet Union are relatively low, and show large differences from one country to another depending mainly upon whether rainfall occurred during the passage of the radioactive cloud.

In the present study, we analyzed two groups of populations - Chernobyl liquidators and people living and working in contaminated by radionuclides territories.
It is generally accepted that the group of Chernobyl liquidators was the most affected population by the accident. A restricted number, of the order of 400 people, including plant staff, firefighters and medical aid personnel, were on the site during the accident and its immediate aftermath, and received very high doses from a variety of sources and exposure pathways.

The doses to these people ranged from a few Gy to well above 10 Gy to the whole body from external irradiation and comparable or even higher internal doses, in particular to the thyroid, from incorporation of radionuclides. The largest group of liquidators participated in clean-up operations for variable durations over a number of years after the accident. Although they were no longer working in emergency conditions, and were subject to controls and dose limitations, they received significant doses ranging from tens to hundreds of millisieverts.

By various estimates, today about several million people still reside in areas that will remain highly contaminated by Chernobyl radioactive pollution for many years to come. There were two main pathways of populations exposure: the radiation dose to the thyroid as a result of the concentration of radioiodine in the gland and the whole-body dose caused largely by external irradiation mainly from radio cesium. The Chernobyl catastrophe released enormous quantities of radioactive iodine, which becomes concentrated in the thyroid glands of exposed individuals. ${ }^{131}$ I leads to pathological cell modifications in thyroid. This process is exacerbated in areas - such as Belarus, southwest Russia and Ukraine - where iodine deficiency is endemic.

The study of the nature and possibility of modification of bystander factors, circulating in blood serum of populations, affected by the Chernobyl accident, will facilitate better understanding of radiation damage mechanisms. Knowledge of these mechanisms is increasingly important for cancer radiation therapy - for using special correcting coefficients taking into consideration effects of bystander factors. The anti-tumor therapy could be more effective and safe. Revealing the nature of the damaging bystander factors will promote more exact dose evaluation and radiation risk for people, exposed to ionizing radiation, as the risk of radiation exposure at low doses, calculated using direct and bystander effects, may be higher as compared to that calculated only from direct effects. The ability to neutralize and modify these factors may help to decrease significantly the effects of whole body radiation exposure.

\section{MATERIALS AND METHODS}

\subsection{Cell culture}

In the present study HPV-G cells (human keratinocytes, immortalized by human papilloma virus transfection), deficient in p53 were used as a test system. Cells were cultured in Dulbecco's MEM: F12 (1:1) medium supplemented with 10\% Fetal bovine serum, $1 \%$ penicillin-streptomycin (1g per $100 \mathrm{ml}$ ), $1 \%$ L-glutamine and $1 \mu \mathrm{g} / \mathrm{ml}$ hydrocortisone. The cells 
were maintained in an incubator at 37 degrees centigrade, with $95 \%$ humidity and $5 \%$ carbon dioxide and routinely subcultured every 8-10 days.

\subsection{Affected populations}

The objects of investigation were blood sera from people affected by the Chernobyl accident: Chernobyl liquidators of 1986-1987 (22 persons), Polessky State Radiation Environmental Reserve workers ${ }^{1}$ (PSRER, 21 persons) and people, living in areas of Gomel region (GR) contaminated by radionuclides (15 persons). The analyzed groups also included four persons with acute virus infection (flu) living in areas contaminated by radionuclides. The control group included clinically healthy people from non-contaminated areas, corresponding to the main group in age and sex (36 persons).

\subsection{Serum extraction}

The blood samples were taken and placed in Vacutainers for serum extraction (Becton Dickinson, USA), centrifuged at $2000 \mathrm{~g}$ for 10 minutes, and the serum was frozen and stored at $-20^{\circ} \mathrm{C}$ before use. Before freezing, the sera were filtered through $0,22 \mu \mathrm{m}$ filters (Nalgene, USA) in order to remove all residual cell components of the blood.

\subsection{Radioprotective substances}

Antioxidant substances melanin and melatonin were used as radioprotectors. Melanin (Belarusian pharmaceutical association, Minsk) was used at 10 $\mathrm{mg} / \mathrm{l}$ concentration, melatonin (Sigma, Germany) - at $10 \mathrm{mg} / \mathrm{ml}$.

\subsection{Micronucleus assay}

After plating, cells were left at $37^{\circ} \mathrm{C}$ in the $\mathrm{CO} 2$ incubator to be attached for 12 hours. The blood serum from affected populations was placed into $25 \mathrm{~cm} 2$ flasks (NUNC, USA) (6000 cells per flask) 1-2 days after plating, and cells were placed again in the incubator for 1-2 h. Then cytochalasin B was added (7 $\mu \mathrm{g} / \mathrm{ml}$ concentration) and the cells were incubated for 24 hours. The cell culture medium was removed, the cells were washed with PBS and fixed with chilled Carnoy's solution ( 1 part of glacial acetic acid and 3 parts of methanol, 10-15 $\mathrm{ml} 3$ times for 10-20 min). Later flasks were dried and stained with $10 \%$ Giemsa solution. The micronuclei count was carried out under inverted microscope. The data are presented as the micronuclei (cells with micronuclei) frequency recorded per 1000 binucleated cells \pm standard error.

\subsection{Alamar Blue assay}

HPV-G cells were plated on 96-well microplates (NUNC, USA) at the concentration of $2 \times 104$ cells/well. After plating, cells were incubated for 24 hours to allow

\footnotetext{
1 Polessky State Radiation and Environment Reserve (founded in 1988) is a territory of Gomel region, where humans cannot live because of the very high levels of radiation contamination. The territory of this reservation is $\mathbf{2 1 5 . 5}$ hectares.
}

attachment to the bottom of the well. Then the medium was removed, cells were rinsed with phosphate buffered saline (PBS) and the blood serum from the Chernobyl accident populations was added to the cells together with melanin and melatonin as appropriate. Microplates were placed into the incubators. Twentyfour hours later, serum was removed, cells were rinsed with PBS and $100 \mu \mathrm{l}$ of a 5\% solution of Alamar Blue prepared in phenol red free DMEM media were added. Microplates were placed again into the incubators. Three hours later, fluorescence was quantified using a microplate reader (TECAN GENios, Grödig, Austria) at the respective excitation and emission wavelength of 540 and $595 \mathrm{~nm}$, respectively Wells containing medium and Alamar Blue without cells were used as blanks. The mean fluorescent units for the three replicate cultures for each exposure treatment were calculated and the mean blank value was subtracted from these results.

\subsection{Statistical analysis}

All experiments were repeated at least three times, and within each experiment, cultures were set up in triplicate. The data are presented as the means \pm standard errors.

When the distribution was normal, significance was determined using the $t$ test. The level of significance was chosen as $95 \%$; at $t \geq 2.67$, the difference is highly significant at $p<0.01$, at $t \geq 1.96$, the difference is significant at $p<0.05$. At $\mathrm{p}>0.05$, the difference is not significant.

The Mann-Whitney $U$ test is a nonparametric alternative to the t-test for independent samples and was calculated using STATISTICA 8.0 (Statsoft, USA). The $U$ statistics is accompanied by a $z$ value (normal distribution variety value), and the respective $P$-value.

\section{RESULTS}

3.1. Study of effects of blood sera from people, affected by the Chernobyl accident on micronuclei frequency in $\mathrm{HPV}-\mathrm{G}$ cells

Table 1 presents data on the effects of blood serum samples from different population groups on the total micronuclei frequency in HPV-G cells (average data presented).

The micronuclei frequency in the controls indicates the level of spontaneous mutagenesis (it is comparatively low). The number of the cells with two and especially three micronuclei was very low as compared with the number of cells with one micronucleus.

As seen from Table 1, people exposed to chronic radiation (PSRER workers) have an increased level of bystander factors, expressed as a considerable increase in micronuclei frequency (almost three times as high as the control level $-248.03 \%$ \pm 20.77 as compared with $80.30 \%$ o13.14, $P<0.01$ ).

At the same time, an increase in the number of the cells with more than one micronucleus was observed. Thus, the data clearly indicate that intensive chronic 
irradiation of PSRER workers significantly promoted accumulation of bystander factors in blood.

Similar results were observed after comparative analysis of the micronuclei frequency between the control group and the liquidators group (people exposed to acute radiation). The total micronuclei frequency of $273.7 \%$ with micronuclei of $235.6 \%$ serum from liquidators were significantly higher than those in the control group (in both cases $P>0.01$ ). In addition, an increase in the number of cells with more than one micronucleus was observed.

Table 1. The effects of blood serum samples from different groups of population on HPV-G micronuclei (MN) frequency (average data are presented)

\begin{tabular}{|c|c|c|}
\hline & $\begin{array}{l}\text { Total number of } \\
\text { cells with MN, } \\
\% \text { \% }\end{array}$ & $\begin{array}{l}\text { Total MN } \\
\text { frequency, \%o }\end{array}$ \\
\hline control & $74,8 \pm 12,4$ & $80,3 \pm 13,1$ \\
\hline liquidators & $235,6 \pm 14,0^{* \dagger}$ & $273,7 \pm 22,4^{* \dagger}$ \\
\hline $\begin{array}{l}\text { PSRER } \\
\text { workers }\end{array}$ & $219,7 \pm 18,3^{* \dagger}$ & $248,0 \pm 20,8^{* \dagger}$ \\
\hline $\begin{array}{l}\text { residents of } \\
\text { GR }\end{array}$ & $143,1 \pm 9,3^{*}$ & $156,5 \pm 11,2^{*}$ \\
\hline residents of & & \\
\hline $\begin{array}{l}\text { GR with acute } \\
\text { virus infection }\end{array}$ & $301,2 \pm 7,8^{* \dagger}$ & $435,6 \pm 8,4^{* \dagger}$ \\
\hline
\end{tabular}

The level of micronucleus frequency induced by serum samples from the residents of contaminated areas of Gomel region is statistically significantly different from the control $(156.47 \% \circ \pm 11.22$ vs. $80.30 \%$ o13.14 $P<0.01)$, but much lower than in people, exposed to additional radiation influence (as compared to liquidators and PSRER workers in all cases $P<0.01$ ).

At the same time, the level of the micronucleus frequency induced by serum samples from the residents with acute virus infection was higher than in all previous cases - the micronucleus frequency induced by the serum from these patients is $435.6 \% \pm \pm 8.4$, and the number of cells with micronuclei is $301.2 \% \pm 7.8$. These figures are much higher than those for liquidators and PSRER workers (in both cases $P<0.01)$.

\subsection{Study of the effects of blood sera from population groups, affected by the Chernobyl accident on metabolic activity of HPV-G cells}

The study was run to understand if there were any bystander factors observed in serum samples of these population groups, which could affect cell metabolic activity. The blood serum samples for Alamar Blue analysis were taken from Chernobyl liquidators and residents of contaminated areas of Gomel region.

Figure 1 presents the average data for all these groups (metabolic activity of intact cells was taken as $100 \%)$.
The viability of the cells treated with serum samples from non-irradiated individuals is very close to intact levels $(t=0.33, \quad P<0.01$ - the difference is not significant). It means that the metabolic activity of these cells was not damaged by serum samples from healthy people, not increasing or decreasing significantly the viability of HPV-G cells.

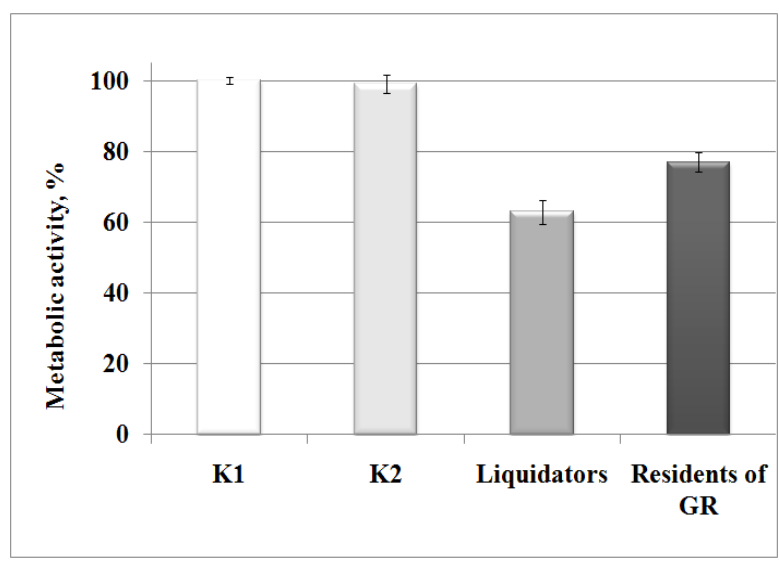

Figure 1. Cytotoxic effects of serum samples from the control groups, Chernobyl liquidators and residents of GR on metabolic activity of HPV-G cells (as a percentage of intact cells, average data for all groups of populations are presented)

Treatment of the cells with serum samples from Chernobyl liquidators clearly reduces the viability of HPV-G cells more than 1.5 times - from $24.89 \pm 0.25 \times 10^{3} \mathrm{FU}$ (intact cells) and $24.67 \pm 0.62 \times 10^{3}$ FU (non-irradiated individuals) to $15.65 \pm 0.82 \times 10^{3} \mathrm{FU}$ (liquidators); $P<0.01$ in both cases $(t=10.79$ and 8.77, respectively). Treatment of HPV-G cells with serum samples from residents of contaminated areas of Gomel region also reduces the viability of cells $\left(19.16 \pm 0.71 \times 10^{3} \mathrm{FU}, t=7.62\right.$ compared to intact cells, $P<0.01$ ), but not as significantly as sera from liquidators.

Table 2 presents the results of the comparison between all groups using the Mann-Whitney U-test.

Table 2. Comparative analysis of 4 independent groups (intact cells $\mathrm{K}_{1}$, non-irradiated individuals $\mathrm{K}_{2}$, liquidators and residents of GR) using Mann-Whitney U-test

\begin{tabular}{|l|c|c|}
\hline \multicolumn{1}{|c|}{ Groups of comparison } & $\mathbf{Z}$ & $\boldsymbol{P}$ \\
\hline $\mathrm{K}_{1}$ vs. $\mathrm{K}_{2}$ & 0,39 & $>0,6$ \\
$\mathrm{~K}_{1}$ vs. Liquidators & $3,92^{*}$ & $<0,00001$ \\
$\mathrm{~K}_{1}$ vs. GR residents & $3,40^{*}$ & $<0,001$ \\
$\mathrm{~K}_{2}$ vs. Liquidators & $3,54^{*}$ & $<0,0005$ \\
$\mathrm{~K}_{2}$ vs. GR residents & $3,07^{*}$ & $<0,005$ \\
Liquidators $v$ s. GR residents & $3,03^{*}$ & $<0,005$ \\
\hline * statistically significant at $\mathrm{p}<\mathrm{0,01}$ \\
\hline
\end{tabular}

The analysis of differences between all groups showed that there was statistically significant 
difference (with at least $P<0.005$ ) between all groups excluding intact cells vs. non-irradiated population group (P>0.05). The highest difference observed between liquidators $v s$. intact cells.

\subsection{Study of the effects of antioxidant substances on bystander factors}

In previous studies [10] we have shown that bystander effect when induced in vitro by transfer of culture medium from irradiated cells to non-irradiated could be decreased using radioprotectors with antioxidant activity (melanin, melatonin). In the present investigation, we have studied the possibility of these substances to neutralize bystander factors, induced in vivo.

Figure 2 presents the results of an attempt to modify the effects of blood serum samples from Chernobyl liquidators on metabolic activity of HPV-G cells using melanin and melatonin (average data).

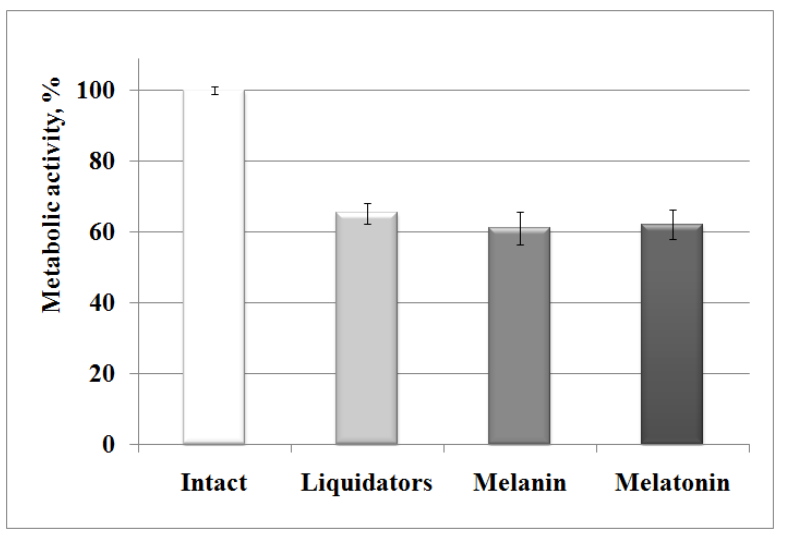

Figure 2. Effects of melanin and melatonin on HPV-G cells treated with blood serum samples from Chernobyl liquidators (control was taken as 100\%)

Figure 2 shows that addition of melanin and melatonin to the medium together with serum samples from Chernobyl liquidators does not have any protective effect. The viability of cells treated with only serum samples $\left(16.24 \pm 0.73 \times 10^{3} \mathrm{FU}\right)$ is almost the same as viability of cells treated with serum samples and melanin $\left(15.21 \pm 1.13 \times 10^{3} \quad \mathrm{FU}\right)$ or melatonin $\left(15.45 \pm 1.04 \times 10^{3} \mathrm{FU}\right)$ - the difference is not significant $(t=0.77$ and $t=0.62$, respectively; $P>0.05$ in both cases).

\section{Discussion}

In similar studies on liquidators from Armenia [7, 11] it was shown that only $42 \%$ of liquidators had an increased level of clastogenic factors (chromosome aberrations) as compared to the level of spontaneous mutations. In our experiments $100 \%$ of liquidators, 95\% of PSRER workers and $82 \%$ of residents from contaminated areas had an increased level of factors as compared to the control levels. A possible explanation of such difference could be the different protocols used in analysis. As mentioned above, HPV-G cells are much more sensitive to mutagenic factors as compared to peripheral blood lymphocytes. Another explanation could be that the cohort from Dr. Emerit studies [12] included liquidators of 1986-1988. After 1987, the dose limits for people engaged in clean-up works significantly decreased. Therefore, liquidators from 1986 expressed higher levels of damaging factors as compared to liquidators of 1987-1988. In the present study liquidators of the consequences of the Chernobyl accident in 1986-1987 were analyzed, which were the most affected group and received the highest doses of radiation (higher than $250 \mathrm{mGy}$ ).

According to the results of the present study, bystander factors persist in the blood of Chernobyl liquidators for more than 20 years after the accident. In previous studies, it was shown that irradiation of blood in vitro at a radiation dose of $500 \mathrm{mGy}$ resulted in significant clastogenic activity and irradiated cells incubated in fresh culture medium continued to produce factors in culture as it was shown in studies [7, 12].

The absence of the protective effect of antioxidant substances can be explained that bystander factors from serum samples persisted in the blood stream for decades, and their nature has no antioxidant component compared to the direct and bystander factors, induced in in vitro experiments.

Thereby, performed studies have shown that blood sera from population groups affected by the Chernobyl accident contain bystander factors, which are able to induce micronuclei and decrease metabolic activity of recipient cells. Pathological processes, such as acute virus infection, could significantly influence the level of bystander factors, increasing their damaging effect. The developed new method of evaluating of effects of damaging factors from blood sera has obvious advantages as compared to the assays that were used in previous studies [7] and may be applied in long-term monitoring of biological consequences of irradiation.

\section{CONCLUSION}

The results of the study of the mechanisms and nature of bystander factors circulating in blood stream of irradiated individuals make a major contribution in understanding non-direct effects of radiation in vivo and allows obtaining of new data on intercellular communications. The new assay of bystander factors analysis in human blood serum samples will help to evaluate health status of affected by the Chernobyl accident populations. As effects of these factors does not depend on the radiation dose, they are especially important for fractionated radiotherapy patients.

Identification of the nature and mechanisms of bystander factors will significantly help in developing effective methods of long-term evaluation of the radiation effects on health status (including NPP workers) and dose estimation in radiotherapy of cancer diseases.

\section{REFERENCES}

1. H. Nagasawa and J.B. Little, "Induction of Sister Chromatid Exchanges by Extremely Low Doses of Alpha- 
Particles," Cancer Res., vol. 52, no. 22, pp.6394-6396, Nov. 1992.

2. B.E. Lehnert and E.H. Goodwin, "A New Mechanism For DNA Alterations Induced by Alpha Particles Such as Those Emitted by Radon and Radon Progeny," Envir. Health Persp., vol.105, suppl. 5, pp.1095-1101, Sep. 1997.

3. E.I. Azzam, S.M. de Toledo, T. Gooding and J.B. Little, "Intercellular Communication is Involved in the Bystander Regulation of Gene Expression in Human Cells Exposed to Very Low Fluencies of Alpha Particles," Rad. Res., vol.15o, no. 5, pp.497-504, Nov. 1998.

4. A. Bishayee, D.V. Rao and R.W. Howell, "Evidence for Pronounced Bystander Effects Caused by Nonuniform Distributions of Radioactivity Using a Novel ThreeDimensional Tissue Culture Model," Rad. Res., vol.152, no.51, pp.88-97, July 1999.

5. C. Mothersill and C. Seymour, "Medium from Irradiated Human Epithelial Cells but not Human Fibroblasts Reduces the Clonogenic Survival of Unirradiated Cells," Int. J. Rad. Biol., vol.71, no.4, pp.421-427, Apr. 1997.

6. K.M. Prise, O.V. Belyakov, M. Folkard and B.D. Micheal, "Studies on Bystander Effects in Human Fibroblasts Using a Charged Particle Microbeam," Int. J. Rad. Biol., vol.74, no. 6, pp. 793-798, Dec. 1998.
7. I. Emerit et al., "Transferable Clastogenic Activity in Plasma from Persons Exposed as Salvage Personnel of the Chernobyl Reactor," J. Canc. Res. Clin. Oncol., vol.120, no. 9, pp. 558-561, Sep. 1994.

8. K. Baverstock and O.V. Belyakov, "Some Important Questions Connected With Non-Targeted Effects", Mutat. Res., vol.687, no. 1-2, pp. 84-88, May 2010.

9. I. Emerit, "Superoxide Generation by Clastogenic Factors," in Free Radicals, Lipoproteins And Membrane Lipids, A. Crastes De Paulet, L. Douste-Blazy And R. Paoletti, Eds., New York (NY), USA: Plenum Press (Springer), 1991, sec. 1, pp.99-104.

10. I. Mosse, P. M. Marozik, C. Seymour and C. Mothersill, "The Effect of Melanin on the Bystander Effect in Human Keratinocytes," Mutat. Res., vol.597, no. 1-2, pp. 133-137, May 2006.

11. I. Emerit et al., "Clastogenic Factors in the Plasma of Chernobyl Accident Recovery Workers: Anticlastogenic Effect of Ginkgo Biloba Extract," Rad. Res., vol.144, no. 2, pp. 198-205, Nov. 1995.

12. I. Emerit et al., "Oxidative Stress-Related Clastogenic Factors in Plasma from Chernobyl Liquidators: Protective Effects of Antioxidant Plant Phenols, Vitamins and Oligoelements," Mutat. Res., vol.377, no. 2, pp. 239-246, July 1997. 